\title{
L'objet, la croyance et le sociologue
}

La sociologie de l'art comme œuvre à faire

\section{Antoine Hennion}

\section{(2) OpenEdition}

\section{Journals}

Édition électronique

URL : http://journals.openedition.org/transposition/1673

DOI : 10.4000/transposition. 1673

ISSN : 2110-6134

Éditeur

CRAL - Centre de recherche sur les arts et le langage

Référence électronique

Antoine Hennion, «L'objet, la croyance et le sociologue », Transposition [En ligne], Hors-série 1 | 2018, mis en ligne le 30 janvier 2018, consulté le 09 février 2020. URL : http://journals.openedition.org/ transposition/1673; DOI : 10.4000/transposition.1673

Ce document a été généré automatiquement le 9 février 2020

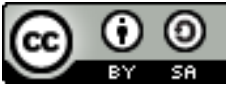

La revue Transposition est mise à disposition selon les termes de la Licence Creative Commons Attribution - Partage dans les Mêmes Conditions 4.0 International. 


\title{
L'objet, la croyance et le sociologue
}

\author{
La sociologie de l'art comme œuvre à faire
}

\section{Antoine Hennion}

Entre musique et sciences sociales, ce volume nous invite à revenir sur le parcours suivi par chacun de nous, à l'échelle d'un séminaire ou d'une vie de recherche. Pour ma part, je vais placer ce retour sous le regard bienveillant de grands auteurs disparus, en repensant à certains qui m'ont marqué - Michel de Certeau ${ }^{1}$ et Louis Marin ${ }^{2}$, au début de mes travaux; plus tard, William James et Étienne Souriau - pour interroger le rapport étrange à son objet qu'entretient la sociologie de l'art. C'est un thème que j'ai sans cesse repris, avec en contrepoint le désir de tirer les leçons que la musique peut donner à la sociologie. C'était le cas avec l'idée de médiation, dans La Passion musicale ${ }^{3}$. Depuis, j'ai abandonné le mot, trop large: la médiation a moins été pour moi un concept théorique qu'une "ethnométhode "; d'un côté, un moyen de relever les mille façons dont historiens sociaux, historiens d'art, et sociologues de l'art ou de la musique établissaient de fait des relations entre leur objet et le social; de l'autre, du côté des musiciens, l'exemple vivant d'un mode opératoire présent dans leur moindre geste, dont ils mettaient ainsi en évidence de multiples aspects.

2 Parler de médiation, c'était sortir la musique de l'opposition entre analyses internes et externes. Il n'y a que des causes partielles, hétérogènes, qu'on ne peut affecter à des registres clairs. À partir de ces causes rassemblées, des effets surgissent de façon en partie imprévisible, toujours à refaire, eux-mêmes irréductibles aux causes qui les ont provoqués. La médiation est un appui nécessaire, elle permet de faire et de faire arriver. Mais elle résiste aussi, elle arrête le cours des choses, elle fait faire autre chose. Certes, c'est plus difficile à mettre en mots simples qu'une relation cause-effet, mais en même temps, si je parle d'ethnométhode, c'est que je ne disais rien là d'ésotérique : le moins musicien des sociologues le comprend, qui a lu le formidable petit livre de Sudnow $^{4}$, sur le musicien qui se met au clavier. Des doigts et des partitions, un apprentissage et des exercices, des genres et des styles, des concerts passés et des médias, mais aussi une situation, sentir l'ambiance, l'auditoire, savoir suivre une idée qui émerge... : tout compte, et dans son détail le plus minutieux. En même temps, rien ne garantit un résultat. Comme souvent, la théorie est en retard sur le sens commun : la 
surprise qui se détache du flux des choses, le sens du geste juste en situation renvoie à l'expérience la plus ordinaire, commune au professionnel et à l'amateur. C'est la passe $\mathrm{du}$ footballeur ou le coup de pinceau du peintre, le plaisir du spectateur ou du buveur, c'est la prise du grimpeur, la blue note du jazzman, le geste de l'aide-soignante, pour prendre des exemples sur lesquels j'ai travaillé.

3 Je ne vais pas reprendre ici la question sur ce plan général, mais sur le plan plus personnel de l'attachement d'un auteur à l'objet auquel il consacre son travail. Une tension naît en effet de "l'amour de l'art » qui (parfois...) habite le sociologue : moteur caché de la recherche, biais subjectif à éviter, ferment indispensable de la compréhension? Un autre récif le fait trembler devant toute analyse de l'art trop proche des termes que l'art tend lui-même: étudier une œuvre ou une pratique artistique, n'est-ce pas d'abord contribuer à la faire art? Qu'il invoque la neutralité axiologique ou le constructivisme pour reculer devant cette compromission ${ }^{5}$, le sociologue ne s'approche de l'art que pour multiplier les preuves qu'il n'est pas pris par lui, au sens des ensorcelés du Bocage ${ }^{6}$, qu'il le traite bien "comme n'importe quoi d'autre ", pour reprendre la célèbre formule de Becker ${ }^{7}$, voire qu'il combat ses prétentions à avoir une réalité propre, selon la conception unilatérale de la perspective critique que Bourdieu a imposée ${ }^{8}$. Étrange résultat: le verbe du sociologue est aussi haut pour critiquer les notions de don, de goût, d'émotion, que son silence est assourdissant dès qu'il s'agit d'approcher un tant soit peu l'expérience qui, selon des formes et des usages infiniment variés, naît au contact des cuvres ${ }^{9} .$. Comment ma discipline pourrait-elle parler de cette présence, lorsque tous les mots qu'elle utilise séparent, isolent, réduisent la part vive des choses, au profit d'une panoplie de structures et de règles, de conventions, de croyances et de déterminations? Comment prendre aussi en compte ce qui se passe, là, irréductible aux conditions qui le permettent?

\section{Un chemin de traverse...}

Mais aborder de front la question des déterminations de mes recherches, c'est risquer de rester trop général sur mes postures sociologiques et trop particulier ou flatteur sur mon auto-analyse. En espérant que cette médiation nous aide à clarifier les choses, je vais introduire un premier passeur dans le jeu: Michel de Certeau. Je vais essayer de «braconner » en compagnie de l'Écriture de l'histoire, pour reformuler à notre attention les questions qu'il a si bien posées à propos de son rapport à la religion, à l'écriture et à l'histoire. Ma «ruse » à moi, pour rester dans son vocabulaire, sera de traduire, de trahir Certeau en musiques, en chant, en vin... Même lui, si austère et peu jouisseur, quel plaisir a-t-il dû éprouver, à coucher avec minutie par écrit, l'une après l'autre, donc en les transformant au moment même où il les re-saisissait, les opérations de pensée qu'il a dû vivre en tant que religieux historien faisant l'histoire de la religion, sous le regard au départ sceptique ou ironique des autres (les religieux le voyant comme un traître, les intellectuels comme un curé). Tous ses thèmes obsessionnels nous ramènent à cette table où il écrit. Sa vie et son œuvre se font écho l'une à l'autre au fur et à mesure qu'elles s'écrivent. Jésuite, aristocrate de province, Certeau s'est fait accepter comme historien tout en gardant son thème spécialisé, le « croire ». Favori de Lacan, il écrit sur la psychanalyse, en particulier sur l'auto-analyse de Freud, qui lui aussi s'analyse en s'écrivant. Il en va de même de ses obsessions les plus théoriques : l'autre, 
la perte, l'absent (Dieu, sa foi, son milieu? qui le saura, pas même lui, sans doute). Il revient sans cesse à l'écriture de l'histoire, saisie comme un faire, une " opérativité ». À la fois productrice de contenu et contenu produit, l'histoire est un présent qui « récupère » le passé (perdu, par définition), en l'écrivant et en l'enterrant dans le même geste.

5 Auto-analyse, donc, chez Certeau, non pas narcissique mais exigeante et rigoureuse, qui ne «remplace » pas le vécu mais le transforme en écrit, à travers des façons de faire : en l'occurrence, l'écriture elle-même, comme activité. Il revient sur sa foi, son Dieu peut-être, l'appartenance à son ordre, tout en les mettant à distance à travers leur écriture. Pour autant, il ne les prend pas pour " objet », il ne prend pas Dieu, sa foi, son passé, etc. pour des objets : jouant un degré de réflexivité en sus, il prend pour objet d'écriture cette opération même qu'est l'écriture, et il met au centre de son écriture les effets de tout écrit par rapport à ce dont il traite. Il montre l'écriture dans le geste même de l'écriture : celui de faire objet son objet (cf. ses belles expressions sur la parole, toujours née de l'absence de ce dont elle parle, mais qu'elle rend présent à partir de ce manque même, et qu'elle fait rentrer dans une histoire). Loin d'appliquer à ce qui le tient comme homme un savoir de savant, sans qu'on puisse faire de lien entre cette motivation initiale, personnelle, et le travail public réalisé ensuite, rendu anonyme sur ce plan par le curieux mécanisme de l'autorité qui mue en rapport de propriété le rapport de production d'un auteur à son œuvre, il déplie à partir de son propre travail et de son propre objet ce qu'est l'écriture comme opération - ou « implication » de soi, comme il dit aussi joliment. Loin de jouer l'indépendance, la distance, il travaille ainsi ce que l'histoire, la religion et l'écriture ont entre-produit, ont produit les unes par et sur les autres, tant dans le récit de la chrétienté et de sa sécularisation moderne, qu'en lui-même.

6 C'est là que je postule (sans preuve) sa jouissance : «alors, qu'en dites-vous, religieux qui croyiez me coincer en montrant que je ne pourrais plus parler de foi avec les outils de la science, savants qui me guettiez pour voir quand je resservirais ma foi après tous ces grands détours pour noyer le poisson et prouver ma compétence professionnelle? Je parle de religion, je parle bien de ma religion, et de ce que c'est que d'en parler - et pour autant, vous n'arriverez pas à disqualifier mon discours, tant du côté de ma foi que du côté de l'histoire. » Il ruse, un mot qu'il aimait : avec ses collègues historiens, avec ses camarades en religion - et aussi avec lui-même, pour rester insituable, être partout et nulle part. Son écriture n'est que ruse avec les places « données », ces places mêmes laissant la place à d'autres, non situées, non situables, infiniment décalées, d'où peuvent seules se dire celles qui s'écrivent. Mais qu'est d'autre l'histoire?

Dieu, que la sociologie paraît positiviste, à côté...

\section{Écrire « à partir de... » ?}

8 Qu'en est-il si, avec mes modestes moyens, j'essaie de transposer ces questions à ma position de sociologue «de " la musique? Un sociologue écrit sur la musique : on se demande aussitôt s'il est musicien, s'il aime la musique. Tel est le lien que je suis allé chercher chez Certeau. Un amour pour la musique dont je sais qu'il ne sera pas mis en mots, ou plutôt qu'il sera toujours autre, un reste, un ailleurs de ce que je pourrais écrire dessus, se trouve néanmoins sans cesse relancé, renforcé, nourri, par une écriture «à partir de» lui. Je pourrais facilement repérer, dans mes propres 
recherches, des moments où j'écris en sociologue, plus "objectivement ", où s'éloigne la musique comme appel, comme "manque » dirait Certeau; et les moments où - sans pouvoir ni prétendre « en " parler - c'est bien ce goût qui me fait écrire. Ce goût dans ce qu'il a d'intraduisible, dans ce qu'il a d'autre, d'étranger à tout écrit, de corporel aussi, ce plaisir intense et incompréhensible. C'est d'ailleurs toujours sur le goût des autres qu'on écrit, même si l'on se prend parfois comme exemple : ce moi-là est un jeu d'écriture. Non pas indépendance, ou substitution, donc, mais passage, écriture réciproque, entre les deux, un présent au nom d'un absent. Sinon, comme le dit Certeau à propos de la religion lorsqu'elle n'est plus que sociologie ou que mystique, parce qu'elle n'est plus que pratique ou que croyance, et que les deux ne sont plus articulées, il n'y a que deux représentations extrêmes, l'une et l'autre vides, desséchées : la musique comme objet donné, la musique comme intimité intransmissible. Cela «marche » au contraire, non pas quand cela s'ajoute ${ }^{10}$, mais quand cela se «trahit », quand chacun est l'autre de l'autre.

Cet entrelacs vu ici du point de vue de l'auteur vaut plus encore si l'on part du point de vue de l'œuvre. Pour indiquer cette piste, au delà d'Umberto Eco ${ }^{11}$ qui, avec les idées d'« œuvre ouverte » et de "lecteur dans le récit ", développe une version sémiologique malicieuse mais linéaire de la présence du spectateur dans l'œuvre, passons par un autre passeur, d'ailleurs ami de Certeau : Louis Marin. Dans Opacité de la peinture ${ }^{12}$, il propose de fascinantes analyses des tableaux de la Renaissance, centrées elles aussi sur la représentation de l'absence comme présence. Aussi éloigné d'une sémiologie formaliste qui les viderait de contenu que d'une analyse positiviste qui les prendrait pour un contenu, il fait des bien nommées Annonciations des montages réflexifs : à partir de leurs supports, elles se constituent en doubles de leur propre message théologique. Ce sont des anges montrant eux-mêmes des anges, pour répéter la présence de l'absence et l'absence du présent. Les peintures sont médiations, à la fois opaques et transparentes, "transitives et réflexives» (p.10): montrer, c'est montrer qu'on montre mais, surtout, montrer qu'on ne montre pas. Marin commente plus loin la représentation des femmes devant le tombeau vide du Christ. « Il n'est plus ici » : « la présence du message [...], affirme l'absence de cet objet, le corps divin, ici et maintenant ; mieux encore, [il en] affirme la présence comme ailleurs et déjà » (p. 126-7, ital. orig.). Comme chez Certeau, loin d'être négatif, l'événement de l'absence, grâce à la peinture, devient récit, sur lequel prend corps une écriture et va se fonder une église. De la théologie en actes que les tableaux réalisent en commentant les textes bibliques, jusqu'aux angles mêmes que font les murs d'église où ils sont accrochés, Marin déploie les couches emboîtées d'œuvres sans auteur ni spectateur, sinon ceux que produit le tableau même, dans l'incertitude de ce qui ne peut être qu'une proposition. La médiation n'est pas une cause; elle ne contient pas ses effets. Elle est passage, elle ouvre "vers... », elle ne détermine pas. Elle conduit et elle doit être conduite, aussi, avec des supports, des gestes et des corps, des outils et des dispositifs. Enfin, elle peut échouer ou réussir, en situation : en ce sens, elle a une effectivité propre, qui dissout l'opposition entre l'œuvre et ses moyens.

10 Je trouve qu'on a rarement écrit sur la peinture de façon aussi musicale ! 


\section{Le point de vue de l'objet}

11 Après ces exemples, on voit mieux à quel point cet objet visé par des pratiques ne pourrait être que manqué, nié ou transformé en enjeu inerte par l'indifférence méthodique du sociologue soi-disant objectif : je dis soi-disant parce que loin d'être une posture objective, il s'agit là d'une façon d'évacuer tous les objets du monde, dans leur résistance, leur opacité, mais aussi et surtout dans leur activité, leur capacité à nous faire ce que nous sommes, pour ne voir en eux que des leurres. Comment nommer cette autre posture, à opposer à une posture neutre, objectivante, où le savant écrit sans laisser de trace de sa présence comme écriveur? Dire qu'elle serait subjective serait bien faible. Alors ? Plus engagée, plus impliquée, plus... croyante, plutôt ${ }^{13}$ ? En tout cas, c'est celle que je vais tenter en parlant musique à partir de mon expérience. Il ne s'agit pas de " parler de moi ». Plutôt de garder en tête, quelque part, cet amour de la musique à la fois comme une sorte de moteur, et comme un impossible enjeu. C'est ce qui fait écrire et ce qui ne peut s'écrire, du moins " complètement ", c'est-à-dire sans aussitôt se faire autre chose. Certeau, lui, bénéficie du double usage du mot histoire, celle que font les acteurs et celle que font les historiens (faire l'histoire/faire de l'histoire, dit-il aussi). Histoire produite, histoire produisante : l'écriture est le rapport entre les deux. Qui est allé aussi loin, en sociologie?

12 Et pourtant, combien le problème s'y pose avec acuité. Combien, en regard de ces nuances infinies des jeux de l'écrit sur quelque chose montrés par Certeau, est insatisfaisante la posture moyenne prenant comme une évidence que le sociologue n'a pas à dire ce qu'est l'art, mais à montrer comment les acteurs " construisent » cette valeur. Dualité bien pauvre, entre essentialisme et constructivisme ${ }^{14}$. Est-il réaliste de voir la sociologie comme un savoir décrivant de l'extérieur le jeu de construction de leurs valeurs par les acteurs? Qui présente encore son goût comme désintéressé, absolu, indépendant de ses origines, au-dessus du jeu de la différenciation sociale? En situation d'entretien avec des amateurs, la sociologie est devenue non seulement un horizon de référence commun à l'intervieweur et l'interviewé, mais l'un des principaux appareillages à travers lesquels l'amateur pense et décrit son propre goût, et celui des autres. Comment tenir compte de cet aspect réflexif inattendu de son activité : le fait que l'indigène lui renvoie désormais, souvent de façon dominante et parfois de façon exclusive, une image qu'elle a elle-même forgée? La solution classique consiste à ajouter à l'analyse de l'objet l'analyse du travail fait par l'observateur sur l'objet, et celle de ses propres prédispositions. Il faut alors remonter toujours plus haut dans une réflexivité conçue comme le fait de se prendre soi-même comme objet. Le danger est double: ouvrir une spirale infinie - où devrait s'arrêter en principe une telle régression? - et, de façon plus dommageable, diriger l'attention plus sur soi-même que sur le monde, les objets et les autres, au risque d'obtenir l'inverse de ce que la critique réflexive voulait faire : de refermer l'observation au lieu d'en élargir la portée.

La solution n'est pas à chercher dans une telle réflexivité du retrait, mais dans une ouverture vers des objets eux-mêmes ouverts. Il faut de l'engagement, de la pénétration, non du recul, pour répondre à la capacité des choses à répondre, à se révéler, à se déployer. Mais c'est bien à ce point que l'expérience de la chose est nécessaire au sociologue. Quand je revois ex post mes thèmes de recherche, la musique comme un faire et non un objet, le goût comme activité (art de faire?) dont l'objet passe par le corps, des dispositifs et des scènes, la situation, les autres; ou encore, sur 
Bach, le fait que c'est lui qui a écrit ce que nous entendons par musique et non la musique qui nous permet de lui donner une place dans son histoire ${ }^{15}$, je me dis qu'une approche certalienne est très suggestive pour parler de musique. «La » musique, on ne sait pas ce que c'est, ou mieux, quand c'est de la musique c'est que ce n'en est déjà plus, que ce que «ça » a fait s'est fait reprendre dans une histoire autre. Ni pour le pygmée attendant d'être initié, ni pour le piétiste écoutant la cantate du dimanche, ni pour le rocker des années 60 découvrant sa génération, il n'y a "la » musique, ni même de musique. Il y a quelque chose à travers lequel corps, notes et collectifs s'écrivent ensemble, que ne peuvent épuiser ni la lecture sociale (partant de groupes donnés), ni la lecture musicale (prenant la musique pour les sons émis). Articuler une pratique et une croyance, disait Certeau. Un amour et un objet aimé ? N'est-ce pas là une voie pour mieux parler de la musique? Ma foi...

\section{Retrouver une posture de croyant?}

14 Cette expression, «ma foi », ne me vient pas pour rien. Si les dieux du Ciel nous ont quittés, ils ne nous laissent pas dans un monde matériel, inerte, avec pour seul horizon un désabus cynique, ou une philosophie humaniste s'efforçant misérablement de le recharger de quelque spiritualité. Du plus petit geste aux choix collectifs les plus lourds de conséquences, ils nous laissent devant des mondes à faire. Les sciences sociales se sont ingéniées à faire de la croyance une sorte d'aveuglement utile, un ciment nécessaire pour faire tenir nos collectifs. Michel de Certeau, ou avant lui William James avec sa Volonté de croire, présentaient de la croyance une tout autre perspective, celle d'une prise sur les mondes à venir, d'un engagement sans garantie ni raison, mais sans lequel ces mondes ne sauraient advenir.

15 Pour ma part, c'est surtout à travers la pragmatique du goût que j'ai réalisé ce déplacement ${ }^{16}$. Ce que montrent les amateurs, ces experts dans l'art de faire venir à eux les différences du monde, c'est bien que les objets ne sont pas « déjà là » munis de leurs propriétés, en attente d'être saisis par une pratique relevant, elle, de la culture ou du social. Ce n'est pas une affaire de valeurs à attribuer à des choses, c'est une affaire d'existence, de présence au monde. Qu'il s'agisse de musique ou de vin, les curieuses pratiques des amateurs ne sont ni un simple apprentissage des propriétés de leur objet de passion, ni un folklore nécessaire pour réchauffer par le collectif des objets a priori froids, neutres, arbitraires. Il faut les faire surgir, ces objets de notre plaisir, avec leurs différences, et nous rendre sensibles à ces différences. Il s'agit de quelque chose comme l'art du «faire exister » : un art que manifestent d'abord les êtres et les choses mêmes ${ }^{17}$. Tout se passe entre, sans sujet ni objet clairement défini, mais ce n'est pas pour cela que la performance qui s'accomplit ne tient pas entièrement à un trait imprévu, à la félicité d'un accent, au succès d'un détail qui change tout. Dans chaque cas, l'extrême précision de ce qui se joue est frappante. La petite différence compte plus que ce sur quoi elle se découpe, dans la sculpture d'une expérience toujours à refaire.

Il ne s'agit donc aucunement d'insister sur la pratique, de remonter de la chose faite à l'acte qui la produit, ce qui laisse intacte la question du « à faire » que posera pour nous Souriau. Au lieu d'accuser encore l'opposition entre le faire et l'être, il s'agit de rendre impossible leur perte de contact. James était conscient du problème : peu emballé par le mot de pragmatisme, voyant le danger d'une confusion avec un éloge de la pratique qui laisserait hors champ son objet, il insistait sur les pragmata, ces choses «dans leur 
pluralité ", en tant qu'elles ne sont pas données ${ }^{18}$. En revenant sur la notion d'agency, mes travaux avec Émilie Gomart puis Geneviève Teil sur les amateurs et leurs attachements ont mis l'accent sur l'ambiguïté que le vocabulaire de l'action introduit, même s'il accorde cette capacité aux choses mêmes ${ }^{19}$. Le goût, c'est se faire aimer, mais aussi se laisser faire. L'observation précise des techniques de l'amateur plaide pour l'abandon radical d'une oscillation entre activité et passivité, au profit d'une sorte de surenchère qui les mêle étroitement, mieux rendue en français par une série de verbes, certes peu élégante, qui accumule réfléchis et doubles infinitifs. Se faire aimer, donc, et laisser arriver ce qui se passe, mais cela ne suffit pas : faire ce qu'il faut pour se laisser prendre, se mettre activement en disposition de se laisser emporter, se couler passivement dans une action en cours, laisser faire son propre geste ${ }^{20} \ldots$ Les techniques des sportifs, des chanteurs, des yogi ou des dégustateurs font toutes écho à ce sentiment. Le passif est très actif et l'actif très passif, ils s'augmentent l'un l'autre, ils n'alternent pas. Mise en disponibilité, relâchement, écoute, tous ces mots sont perpendiculaires à l'axe actif/passif, comme l'est le mot passion lui-même, que personne n'entend comme impliquant de la passivité.

C'est la condition pour que les objets fassent de même, qu'ils se déploient, s'expriment, développent leurs arômes, leurs différences, leur présence aux sens de leur appréciateur. À l'instar de ce que nous venons de dire des humains, il faudrait parler de la patience des choses, en plus de leur agency. Le concept d'agencement redistribue avec bonheur dans la méticulosité des dispositifs, des corps et des objets ce que le vocabulaire de l'action sépare ${ }^{21}$. Mais il appellerait peut-être un double, une expression comme le patiencement... Le mot n'existe pas, dommage : il suggérerait mieux l'attention aux choses du monde et la sollicitude de tous les autres êtres par lesquelles ces choses sont aidées à être, comme Souriau lui-même nous aidera à les développer.

18 Au point où nous sommes, je vais donc risquer un ton encore plus personnel. Face à la frilosité d'une conception scientiste (plus que scientifique) de l'enquête sociologique, y a-t-il moyen de parler d'expériences plus intérieures sans verser dans une introspection subjective qui ne serait que l'inversion complaisante de cet objectivisme impuissant à rendre compte de ce que l'art fait vivre? Premier test : peut-on faire un meilleur compte rendu de l'expérience du chanteur en s'appuyant non sur ses impressions " vécues ", mais sur un suivi quasi matériel de ce qui se passe pendant une répétition? Pour ce faire, James, le troisième passeur dans ce texte, apportera un concours décisifif ${ }^{22}$. Second test : toujours en m'appuyant sur des ontologies ouvertes et pluralistes pour rendre compte de l'expérience musicale au delà d'une opposition entre description extérieure et introspection intérieure, je reviendrai sur un entretien avec un improvisateur de jazz. Ce thème nous conduira enfin à la puissante conception de l'« œuvre à faire » qu'Étienne Souriau a élaborée ${ }^{23}$.

\section{Test 1. Les leçons du chant}

Pourquoi James, d'abord? Son principal effort vise à nous sortir d'un dualisme paralysant. Si l'on suppose un monde stable, espace euclidien dans lequel les choses obéissent à leurs lois, alors ne peut lui faire face que la subjectivité humaine, inversement dotée d'un pouvoir de création démiurgique. Mais qu'en est-il si l'on suppose que le monde n'est pas clos, fini ? Chaque réalité en se déployant se fait de plus en plus particulière, au fur et à mesure qu'elle se sculpte elle-même. Je trouve que c'est 
une belle façon de formuler le projet pragmatiste : accompagner le cours d'extensions indéfinies. S'il n'y a que des flux et des expériences toujours en cours, dans un plurivers ouvert et indéterminé, comme dit James ${ }^{24}$, si ces mondes "still in process of making ${ }^{25}$ " sont des réseaux hétérogènes, sans extérieur, en extension ou en rétraction, le problème s'inverse. Tout acte fait être (ou n'y parvient pas), c'est le maintien d'un peu de stabilité qui est l'exception. Réciproquement, sans se réduire à elles, tout acte s'appuie lui-même sur un empilement de choses qui "font faire »: médiations, habitudes, dispositifs, autant de "faire faire " de tout ordre, sans le soutien desquels rien ne se fait. Le «plurivers » de James dessine une topologie qui, me semble-t-il, peut servir de cadre à une auto-description qui ne soit pas le simple " ressenti ", comme on dit à la radio, d'un événement extérieur. Ce n'est pas affaire de psychologie, c'est de l'ontologie, y compris au sens de la physique. Du « vécu », si l'on veut : mais celui de la chose même.

Rentrons donc dans la peau d'un chanteur amateur. J'en parle à la troisième personne, sans cacher que je parle de mon expérience : mais la mettre en mot, c'est déjà en faire autre chose. En particulier, faire le lien avec d'autres pratiques amateurs, sur lesquelles j'ai enquêté. Partons de là, justement, de l'amateur compris comme celui qui fait exister un objet pour lui. Formule insuffisante : elle met l'accent sur l'amateur, alors que personne ne sait mieux que lui qu'au moins autant, c'est l'objet qui le fait exister. Travail artistique, danse, chant, entraînement du sportif : dans leur exercice, chacun avec leurs mots, cette idée revient toujours qu'il faut laisser arriver les choses, le corps, les sons, les gestes ; que viser le résultat l'empêche (" ne cours pas », « attention, tu joues des notes ! »...). Le résultat vient de surcroît, à l'inverse aussitôt barré si l'on veut décider au lieu d'accompagner ce qui se produit. Toutes ces pratiques travaillent le «moment », ce sont des expériences sur l'expérience. Entre-deux, cours des choses, cela ne veut pas dire flou artistique, zone imprécise où tout convient, bien au contraire.

Le chanteur n'est pas dans un flot indéfini. Le lent apprivoisement de sa voix n'a rien d'une technique froide sur laquelle il grefferait son émotion pour lui donner un peu de chaleur. Du corps à l'œuvre qu'il interprète, il n'est pas emporté dans un tumulte vital sans bord ni fond. Il est confronté à des surfaces d'une extrême précision. Il ne parle que d'appuis, de pressions, de projections. Le flux n'est pas le flou. Mais ici, le pluriel est important : des surfaces. Derrière une couche, il y a toujours une autre couche. Le diaphragme, le ventre, le palais, les fosses nasales, qu'il apprend peu à peu à sentir : c'est qu'il ne s'agit pas soit des organes physiologiques, soit de pures images mentales, mais d'une couche d'expérience qui prend consistance. De même pour le souffle, le son, la voix, il n'est pas un de ces éléments qui n'apparaisse sous ces auspices, d'une zone de résistance dont il faut avoir fait l'expérience, qui à la fois émerge, donne des prises, des appuis, et permette de renvoyer vers une autre couche.

Chacune rend l'autre muette : une fois le son mieux maîtrisé par la respiration, par exemple, il n'occupe plus le même lieu, tant dans l'esprit que dans le corps. Ce n'est plus cette matérialité de l'air faisant le lien entre les parties du corps qui le produisent et le son émis, cela devient une sorte de nappe jetée devant soi, qui s'élargit ou se concentre, qui roule sur elle-même ou emporte tout. S'agit-il là de 
formules imaginaires, ou de réalités palpables? De quelle pauvre façon une telle opposition réduirait ce qui se passe ! Certes, le professeur renvoie de telles images à l'élève, mais c'est pour l'aider dans les va-et-vient où celui-ci ne cesse d'osciller, entre l'impression de faire quelque chose, et à l'inverse celle de se laisser faire par une sorte de prise de possession par son propre chant, qu'il n'a « plus » qu'à entretenir, à contrôler sans tenter de le maîtriser - ce qui, loin de le renforcer, en bloquerait aussitôt l'élan.

Dès qu'il s'agit d'avancer, une nouvelle frontière se dessine. Mais une frontière avec quoi ? Nous voici revenus devant l'œuvre? Oui, mais pas au sens d'un grand saut dans le sublime : ce que j'ai décrit pour le souffle ou le son vaut à chacun des seuils à franchir. La musique n'est pas plus donnée que le corps ou la voix, elle aussi prend la forme de couches successives, qu'il faut faire apparaître une à une. Les « mêmes » notes, sur la même feuille de papier, ne sont pas la même chose lorsqu'il faut les déchiffrer, les faire entrer dans son oreille, les associer à des mots, s'habituer à une musique; et lorsqu'ensuite, cette musique qu'on croit encore lire est en réalité déjà là, en soi, et que cet état en fait un être tout autre. Plus tard, les choses s'inverseront plus radicalement encore, entre ce qui semblait si ferme, la partition devant soi, et ce qui semblait s'échapper en tout sens, les efforts désordonnés pour la réaliser en se battant avec les notes, sa voix, son souffle, le son, l'expression. Car au fur et à mesure que la partition est mieux sue, l'œuvre s'en écarte. La surface plane du papier n'est plus qu'un indicateur familier, qui donne quelques repères, et à nouveau, tout reste à faire. Il s'agit moins de " passer » à la musique une fois les notes acquises, comme s'il y avait la technique d'un côté et l'art de l'autre, que de laisser peu à peu prendre consistance ce qu'on n'appelle œuvre que par commodité, qui ressemble plus à une série d'ouvertures : parfois les notes, les accords prennent une réalité quasi physique, comme une statue qu'on sculpterait, et les mots ne sont que sa glèbe ; un autre jour, une phrase se met à vivre, que ses propres notes avaient cachée jusque là ; ou un écho entre paroles et mélodie se met à sonner juste, et une émotion inconnue surgit, au delà de toute musique; ou inversement le chant se fait corps, timbre, vocalité, performance, c'est l'œuvre notée qui n'est plus que le support de ces transports. Chaque fois, une nouvelle surface de résistance prend corps, sur laquelle on ne peut prendre appui qu'en la constituant, de sorte qu'on ne saura jamais qui a fait l'autre. L'idée de couches est celle-là, à condition de ne pas suggérer de progression cumulative, ni même de hiérarchie : plutôt des effets de voiles successifs que, comme au théâtre, un éclairage ferait tour à tour ressortir, chacun d'eux semblant simplement donner sa matière à celui qui se détache...

\section{Test 2. On the Cutting Edge ${ }^{26}$, l'improvisation du jazzman}

L'idée de l'« œuvre à faire » de Souriau ${ }^{27}$ est très différente de ce qu'on peut entendre par là si l'on rabat trop vite l'expression sur des formules voisines, comme celle d'œuvre ouverte, ou inachevée, laissant le lecteur remplir les vides qu'elle tend ${ }^{28}$, le regardeur « faire » l'œuvre d'art (Duchamp), ou le spectateur participer à la création à travers les actes sollicités, programmés ou aléatoires requis de lui par les happenings, 
les installations, les arts "praticables »" ${ }^{29}$. Ce qu'on dit alors, c'est que l'œuvre doit être appropriée, complétée, transformée, qu'elle est un dispositif de captation qui contient et appelle déjà son récepteur, et qu'elle n'est rien sans lui. C'est mieux que de la prendre pour un objet fixe, surtout si cette idée est appliquée aussi aux œuvres traditionnelles. Mais dans cette optique, pour être incomplète, «à terminer ", l'œuvre d'art n'en est pas moins déjà œuvre: elle est moins «à faire " qu'au contraire programmée pour inclure par avance ses possibles. Il faut prendre la mesure de l'immense écart entre ces thèses qu'on peut dire attributionnistes, et la question de l'œuvre à faire telle que Souriau la fait émerger. Son propos n'est pas de redistribuer les cartes pour faire du lecteur, du regardeur ou de l'auditeur un coproducteur de l'œuvre qu'il actualiserait en la recevant. Le point est beaucoup plus radical. L'œuvre est «à faire », non qu'il faille développer l'œuvre existante, ou actualiser les fameuses « intentions » du créateur ${ }^{30}$, mais parce qu'il faut faire advenir ce qui n'est pas : c'est l'œuvre qui appelle à l'aide pour arriver à l'existence, ou plus précisément, à plus d'existence.

21 À l'opposé de l'idée de création, l'homme qui « crée » quelque chose ne part pas de rien. C'est pourquoi Souriau parle d'instauration : l'artiste n'est pas le principe et la source de sa création, il reçoit aide et appui de l'œuvre elle-même; Souriau nomme « ange de l'œuvre » la forme spirituelle qui le sollicite et le questionne ${ }^{31}$. Voilà qui déstabilise le sociologue dressé à montrer comment les hommes font les choses, non comment les choses font les hommes. Lui parle de ce qui fait l'art, de ses conditions, de ses effets, de son institution, de sa pratique, non de ses œuvres (ni, surtout, de son œuvre). Institution de l'art, ou instauration de l'œuvre: on voit que même l'expression plus pragmatique "ce que fait l'objet » comporte le risque d'une réduction de l'objet à ses effets, risque qu'évacue radicalement la proposition de Souriau. C'est le passage qu'il aide à franchir, d'une théorie de l'action à une ontologie d'êtres toujours "à faire ».

Nous situer dans l'espace ouvert par l'idée d'« œuvre à faire » de Souriau peut-il aider à entendre l'expérience de l'improvisateur?

L'improvisation, au sens précis que les musiciens donnent à cette forme, est une performance : a priori, elle a tous les traits de l'œuvre à faire. Mais loin de fournir la solution miracle à nos interrogations, le mot contient toutes les ambivalences que nous cherchons à dénouer. Il les accentue même, entre les avocats d'une liberté de l'instant et les tenants d'une analyse qui réduit ces prétentions à la combinaison d'éléments connus et à l'application de schémas préconçus, longuement répétés jusqu'à ce que, comble de l'illusion, ils semblent couler de source : « Je n'aime pas les formules comme quoi l'impro c'est l'instant, et toujours ces banalités avec d'un côté le travail, les gammes, et puis hop... ", me dit ce saxophoniste qui, tard après un concert, exprimait à mon endroit sa vive irritation contre le discours tout fait sur l'improvisation en jazz : dans un style fort différent, ses formules font directement écho à celles de Souriau. Le débat est balisé, prévisible, tant qu'on ne sort pas d'une telle opposition binaire entre spontanéité et automatisme : "Moij'aime le jazz à cause de ça, qu'on ait un cadre bien plus... la palette des accords et des gammes, le style... c'est ça qui lasse du jazz certains mais moi pas du tout, au contraire c'est ce côté creuser toujours le même sillon et qu'il soit toujours plus intense qui m'excite. » 
L'improvisation appelle à une théorie de l'action située, distribuant son efficacité entre l'entraînement des corps, le recours à des astuces et repères mnémotechniques, l'appui sur les ressources et les affordances qui se présentent associations, réactions de l'instrument, idées qui surgissent - et enfin la capacité de l'acte à se dépasser lui-même ${ }^{32}$. Médiations nécessaires, et toujours insuffisantes à garantir le succès : l'impro est performance à la fois au sens français du mot, qui la rapproche d'un exploit sportif, et au sens anglais qui insiste plus sur la mise en scène de soi et l'effet auto-réalisateur de l'accomplissement d'un rôle qui, même connu, doit être chaque fois revécu et reconnu - ce qui introduit aussi à sa dimension publique. L'improvisation a décidément bien des vertus, à commencer, pour nous, par celle de jouer au plus près d'une frontière subtile, qui passe entre le «faire » et le «faire être », du côté de l'œuvre, et entre le « faire » et le « se laisser faire ", du côté de l'instrumentiste : «Moi, l'image de l'acquis et puis du saut dans le vide ne me plait pas du tout... ce n'est pas ça, on ne coupe rien, c'est l'inverse, je ne vois que des liens moi au contraire, plein de fils qui nous tiennent et nous poussent, plutôt... d'abord on ne l'a pas du tout l'acquis, il faut le faire arriver justement ! On suit son propre mouvement, on le lance et on le suit en même temps, c'est comme des couches qu'on ajoute, il y a des vagues et puis ça pousse, on y va, on trouve des tas de trucs, ça va, et puis ça s'essouffle, c'est qu'on recharge, on rapporte des couches, là c'est vrai on sent qu'on se sert plus des trucs acquis ou mieux, des fois, juste qu'on les laisse arriver, qu'on sent qu'il en vient... ça se reforme, on voit un tour, on le répète, c'est reparti, un peu comme s'il y avait des avancées et des retraites de densité... voilà, ça je vis ça dans l'impro!»

Qu'ajouterait à ces vertus de l'improvisation une formulation attentive à l'idée rigoureuse que Souriau se fait de l'œuvre à faire ? Eh bien, justement, comme y invite de façon paradoxale ce jazzman, la possibilité de plus nettement nous détacher du point de vue du "faiseur ", de celui qui improvise, voire même de l'auditeur qui se laisse prendre, pour insister sur le point de vue de la consistance de l'œuvre produite ainsi, dans le souffle : «L'impro ce n'est pas jouer, c'est jouer qu'on joue, comment dire, mettre en scène soi-même le fait que ça joue ou non, plus ou moins, régler ça en même temps qu'on joue... plus suivre ce qui arrive que le provoquer. »Ce n'est pas une statue, qu'on peut laisser reposer et reprendre plus tard, c'est vrai ; mais ce point est secondaire par rapport à l'idée centrale de l'improvisation : certes, se laisser emporter, basculer dans les moments de débordement que permet l'élan depuis les cadres, les ficelles, les entraînements - oui, mais vers où, et surtout, à l'appel de quoi ${ }^{33}$ ? La question de l'improvisation semble n'être toujours posée qu'à moitié, depuis ses producteurs (disons ses coproducteurs, en incluant ses spectateurs et ses dispositifs). La poser aussi du point de vue de l'œuvre lui donne une tout autre profondeur : «C'est comme les acteurs, avec une expression ils trimballent tout avec eux, tout le théâtre, toute leur histoire, tout leur corps, et c'est de ça qu'ils jouissent, et c'est pour ça qu'on en jouit... nous on a aussi un "texte", même si ce n'est pas écrit, on suit un objet qui est hors de notre corps, notre matériau c'est toute la musique... »

23 Cette impro - non pas l'improvisation en général mais celle-ci, là - a-t-elle apporté plus qu'une œuvre mille fois retravaillée, une présence de la musique qu'aucune œuvre interprétée n'aurait pu atteindre ? À défaut de quoi, elle n'aurait fait que profiter de l'effusion du direct, elle aurait dissimulé son état d'esquisse incomplète, au lieu de l'affronter. Question chaque fois ouverte... Que dit d'autre Souriau? Nous sommes 
moins appelés à coproduire une œuvre qu'il faudrait terminer qu'à aider une œuvre qui se crée elle-même à achever son accomplissement. Processus étrange - mais en même temps, même pour produire l'œuvre la plus modeste, ce sentiment est familier à quiconque se bat avec une plume, un ciseau ou un pinceau. Souriau l'énonce de façon impitoyablement rigoureuse : si l'œuvre s'auto-crée (c'est ce qui la fait œuvre, ce qui peut même, éventuellement, promouvoir son existence en être, si elle atteint assez de consistance ${ }^{34}$ ), pour autant, elle n'en a pas moins besoin pour cela non seulement de son créateur, mais de nous tous. Il évoque « cette responsabilité [...], qui nous incombe à propos de tout l'inachevé du monde » : « le moindre nuage, la plus petite fleur, le plus petit oiseau, une roche, une montagne, une vague de la mer ", tout fonctionne sur ce mode d'un appel à plus d'existence, faisant dépendre de la sollicitude de chacun l'existence de tous ${ }^{35}$.

Si Souriau appelle notre discipline à la modestie, il lui dessine aussi une ambition qui soit à la hauteur de ses enjeux : comme toute œuvre, la sociologie de l'art reste "à faire ».

\section{BIBLIOGRAPHIE}

BECKER, Howard S., Les Mondes de l'art, Paris, Flammarion, 1988.

BOURDIEU, Pierre, La Distinction, Paris, Minuit, 1979.

BOURRIAUD, Nicolas, Esthétique relationnelle, Dijon, Les Presses du réel, 1998.

CALLON, Michel, «Qu'est-ce qu'un agencement marchand? », CALLON, M. \& al. (eds), Sociologie des agencements marchands, Paris, Presses de l'École des Mines, 2013, p. 425-426.

CERTEAU, Michel de, « Croire : une pratique de la différence », Documents, Centro Internazionale di Semiotica e di Linguistica, Università di Urbino, 106-A, 1981, p. 1-21.

CERTEAU, Michel de, L'Écriture de l'histoire, Paris, Gallimard, 1975.

CHATEAU, Dominique, L'Art comptant pour un, Dijon, Les Presses du réel, 2009.

DANTO, Arthur, La Transfiguration du banal. Une philosophie de l'art, Paris, Le Seuil, 1989.

ECO, Umberto, L'Euvre ouverte, Paris, Le Seuil, 1965.

ECO, Umberto, Lector in fabula, Paris, Grasset, 1985.

FAUQUET, Jean-Marie et HENNION, Antoine, La Grandeur de Bach, Paris, Fayard, 2000.

FAVRET-SAADA, Jeanne, Les Mots, la mort, les sorts. La sorcellerie dans le bocage, Paris, Gallimard, 1977.

FOURMENTRAUX, Jean-Pierre (ed.), L’ère post-média, Paris, Hermann, 2012.

GENETTE, Gérard, L'Euvre de l'art, Paris, Le Seuil, 1994-1997. 
GOMART, Émilie et HENNION, Antoine, « A Sociology of Attachment: Music Amateurs, Drug Users ", LAW, John et HASSARD, John (eds), Actor Network Theory and After, Oxford, Blackwell, 1999, p. 220-247.

GROSSEIN, Jean-Pierre, « Max Weber “à la française" ? De l'urgente nécessité d'une critique des traductions ", in Revue française de sociologie, 46/4 (2005), p. 883-904.

HENNION, Antoine, « “Aussi vite que possible...” La virtuosité, une vérité de la performance musicale ? ", Ateliers d'anthropologie du LESC 35, 2011, http://ateliers.revues.org/8764.

HENNION, Antoine, « D'une sociologie de la médiation à une pragmatique des attachements », SociologieS, 2013, http://sociologies.revues.org/4353

HENNION, Antoine, « Enquêter sur nos attachements. Comment hériter de William James?», SociologieS, 2015, http://sociologies.revues.org/4953

HENNION, Antoine, « Jouer, interpréter, écouter. Pratiquer la musique, ou la faire agir ? », FOURMENTRAUX, Jean-Pierre (ed.), L'Ère post-média, Paris, Hermann, 2012, p. 87-102.

HENNION, Antoine, « La mémoire et l'instant. Improvisation sur un thème de Denis Laborde ", Tracés 18 (2010), p. 141-152.

HENNION, Antoine, « Paying Attention : What is Tasting Wine About? », Moments of Valuation. Exploring sites of dissonance, BERTHOIN ANTAL, Ariane, HUTTER, Michael et STARK, David (eds), Oxford, Oxford University Press, 2015, p. 37-56.

HENNION, Antoine, « Pragmatics of taste ", JACOBS, M. et HANRAHAN, N. (eds), The Blackwell Companion to the Sociology of Culture, Oxford, Blackwell, 2004, p. 131-144.

HENNION, Antoine, « Those Things That Hold Us Together: Taste and Sociology », Cultural Sociology 1/1 (2007), p. 97-114.

HENNION, Antoine, « Vous avez dit attachements ?... », Débordements. Mélanges en l'honneur de Michel Callon, AKRICH, M. \& al. (eds), Paris, Presses de l'École des Mines, 2010, p. 179-190.

HENNION, Antoine et MONNIN, Alexandre, « Sous la dictée de l'ange. Enquêter sous le signe d'Étienne Souriau ", Étienne Souriau. Une ontologie de l'instauration, COURTOIS-L'HEUREUX, Fleur et WIAME, Aline (eds), Paris, Vrin, 2015, p. 131-156.

HENNION, Antoine, La Passion musicale, Paris, Métailié, 2007 [1993, trad. angl. 2015].

ISER, Wolfgang, L'Acte de lecture, Bruxelles, Mardaga, 1985.

JAMES, William, Essais d'empirisme radical, Marseille, Agone, 2005 [1912].

JAMES, William, La Volonté de croire, Paris, Les Empêcheurs de penser en rond/Le Seuil, 2005 [1897].

JAMES, William, Philosophie de l'expérience. Un univers pluraliste, Paris, Les Empêcheurs de penser en rond/Le Seuil, 2007 [1909].

JAMES, William, The Meaning of Truth, New York, Longmans, Green \& Co, 1909.

JAUSS, Hans R., Pour une esthétique de la réception, Paris, Gallimard, 1978.

KALINOWSKI, Irène, « Leçons wéberiennes sur la science et la propagande », introduction à WEBER, Max, La Science, profession et vocation, Marseille, Agone, 2005.

LABORDE, Denis, La Mémoire et l'instant. Les improvisations chantées du bertsulari basque, Bayonne/ Donostia, Elkar, 2005. 
LAPOUJADE, David, «Étienne Souriau. Une philosophie des existences moindres », in DEBAISE, Didier (éd.), Philosophie des possessions, Dijon, Les Presses du Réel, 2011, p. 167-196.

LATOUR, Bruno, Enquête sur les modes d'existence, Paris, La Découverte, 2012.

MARIN, Louis, Opacité de la peinture, Paris, Usher, 1989 (rééd. Paris, Éditions de l’EHESS, 2006).

MICHAUD, Yves, L'Art à l'état gazeux, Paris, Stock, 2004.

SOURIAU, Étienne, «Du mode d'existence de l'œuvre à faire », Bulletin de la Société française de philosophie, 50/1 (1956), p. 4-24 (rééd. SOURIAU, Étienne, Les différents modes d'existence, I. Stengers et B. Latour prés., Paris, PUF, 2009, p. 195-217).

SOURIAU, Étienne, L’Ombre de Dieu, Paris, PUF, 1955.

STENGERS, Isabelle, « William James : une éthique de la pensée ? », DEBAISE, Didier (éd.), Vie et expérimentation. Peirce, James, Dewey, Paris, Vrin, 2007, p. 147-174.

SUDNOW, David, Ways of the hand: the organization of improvised conduct, Cambridge MA, Harvard University Press, 1978.

TEIL, Geneviève et HENNION, Antoine, « Discovering Quality or Performing Taste? A Sociology of the Amateur ", HARVEY, M., McMEEKIN, A. et WARDE, A. (eds), Theoretical Approaches to Food Quality, Manchester, Manchester University Press, 2004, p. 19-37.

TEIL, Geneviève et HENNION, Antoine, Le Vin et l'environnement, Paris, Presses de l'École des Mines, 2011.

\section{NOTES}

1. Revenu des États-Unis, mais n'ayant pas de poste à l'université ni à l'EHESS, il a été accueilli un an au CSI, où tout jeune, j'avais dû discuter en sa présence son texte «Croire : une pratique de la différence » (1981), que j'avais rapproché du cas du renouveau de la musique baroque. Je me souviens encore de l'expérience...

2. Peu avant sa mort précoce, nous l'avions invité discuter au séminaire du CSI de l'Opacité de la peinture (1989). Je reviens plus bas sur ce livre exceptionnel.

3. HENNION, Antoine, La Passion musicale, Paris, Métailié, 2007 [1993, trad. angl. 2015].

4. SUDNOW, David, Ways of the hand: the organization of improvised conduct, Cambridge MA, Harvard University Press, 1978.

5. Comme le rappellent en vain ses commentateurs, la célèbre mise en garde de Weber ne consiste pas à exiger du sociologue qu'il s'en tienne aux faits et ne formule pas de jugements de valeur. Il dit l'inverse : faits et valeurs étant inséparables, son devoir est d'expliciter et d'afficher ses valeurs, donnant ainsi la possibilité d'en choisir d'autres, au lieu de les dissimuler sous des énoncés neutres, se prétendant factuels. I. Kalinowski préfère ainsi parler de «non-imposition de valeurs" (KALINOWSKI, Irène, "Leçons wéberiennes sur la science et la propagande ", introduction à WEBER, Max, La Science, profession et vocation, Marseille, Agone, 2005), tandis que, discutant la traduction de Gesinnung par disposition, J.-P. Grossein souligne avec raison les parentés entre Weber et le pragmatisme (GROSSEIN, Jean-Pierre, "Max Weber "à la française" ? De l'urgente nécessité d'une critique des traductions », Revue française de sociologie 46/4 (2005), p. 883-904).

6. Étudiés par J. Favret-Saada dans un ouvrage qui a fait date : FAVRET-SAADA, Jeanne, Les Mots, la mort, les sorts. La sorcellerie dans le bocage, Paris, Gallimard, 1977.

7. "Treating art as not so very different from other kinds of work" (Art Worlds 1982: ix-x ; traduction française : BECKER, Howard S., Les Mondes de l'art, Paris, Flammarion, 1988). 
8. BOURDIEU, Pierre, La Distinction, Paris, Minuit, 1979.

9. L'œuvre de l'art, et non l'œuvre d'art, selon la belle formulation de G. Genette (L'CEuvre de l'art, Paris, Le Seuil, 1994-1997) : c'est-à-dire travail en cours, mise en mouvement, « faire faire » qui ne se saisit nulle part ailleurs que dans la série infinie des relances et des reprises. Je reviendrai sur ce thème avec Souriau.

10. Pour parler sans en parler de ces objets impliquants, les auteurs en sciences sociales manient avec virtuosité l'art de la préface, où livrer des considérations personnelles, avant de poursuivre avec leur lecteur anonyme un travail lui aussi anonyme, exempté de ces considérations.

11. ECO, Umberto, L'CEuvre ouverte, Paris, Le Seuil, 1965 ; Lector in fabula, Paris, Grasset, 1985.

12. MARIN, Louis, Opacité de la peinture, Paris, Usher, 1989 (rééd. Paris, Éditions de l'EHESS, 2006).

13. Le mot est sans doute le bon, à condition qu'on le prenne au sens où James réhabilite la croyance comme rapport nécessaire, premier, au monde, y compris dans la démarche scientifique, dans La Volonté de croire (JAMES, William, La Volonté de croire, Paris, Les Empêcheurs de penser en rond/Le Seuil, 2005 [1897]).

14. HENNION, Antoine, "D'une sociologie de la médiation à une pragmatique des attachements ", Sociologies [en ligne], $2013 \mathrm{http}: / /$ sociologies.revues.org/4353. Sur l'origine de la sociologie comme théorie de la croyance, voir HENNION, Antoine, La Passion musicale, op. cit., p. 35-65.

15. FAUQUET, Jean-Marie et HENNION, Antoine, La Grandeur de Bach, Paris, Fayard, 2000.

16. HENNION, Antoine, "Pragmatics of taste ", JACOBS, M. et N. HANRAHAN (eds), The Blackwell Companion to the Sociology of Culture, Oxford, Blackwell, 2004, p. 131-144.

17. C'est la force du mode [REP] proposé par Latour dans son enquête sur les modes d'existence (2012).

18. JAMES, William, The Meaning of Truth, New York, Longmans, Green \& Co, 1909, p. 210.

19. GOMART, Émilie et HENNION, Antoine, «A Sociology of Attachment: Music Amateurs, Drug Users ", LAW, John et HASSARD, John (eds), Actor Network Theory and After, Oxford, Blackwell, 1999, p. 220-247 ; TEIL, Geneviève et HENNION, Antoine, «Discovering Quality or Performing Taste? A Sociology of the Amateur ", HARVEY, M., McMEEKIN, A. et WARDE, A. (eds), Theoretical Approaches to Food Quality, Manchester, Manchester University Press, 2004, p. 19-37 ; TEIL, Geneviève et HENNION, Antoine, Le Vin et l'environnement, Paris, Presses de l'École des Mines, 2011 ; HENNION, Antoine, "Vous avez dit attachements ?... ", Débordements. Mélanges en l'honneur de Michel Callon, AKRICH, M. \& al. (eds), Paris, Presses de l'École des Mines, 2010, p. 179-190.

20. HENNION, Antoine, "Those Things That Hold Us Together: Taste and Sociology ", Cultural Sociology 1/1 (2007), p. 97-114.

21. Comme le défend Callon en le reprenant à Deleuze, le mot est bien préférable à celui d'assemblage, inerte et indifférencié. (CALLON, Michel, «Qu'est-ce qu'un agencement marchand?", CALLON, M. \& al. (eds), Sociologie des agencements marchands, Paris, Presses de l'École des Mines, 2013, p. 425-426.) Jouer une sonate, courir un 100 mètres, cuisiner, soigner des malades ou faire fleurir un parc, c'est indéfiniment sculpter une multitude hétéroclite de mille détails qui chacun permettent aux autres de mieux saillir, pour leur servir à leur tour de prises ce que suggère mieux le mot agencement.

22. STENGERS, Isabelle, «William James : une éthique de la pensée ? », DEBAISE, Didier (éd.), Vie et expérimentation. Peirce, James, Dewey, Paris, Vrin, 2007, p. 147-174 ; HENNION, Antoine, «Enquêter sur nos attachements. Comment hériter de William James?", Sociologies [en ligne] (2015), http://sociologies.revues.org/4953

23. SOURIAU, Étienne, « Du mode d'existence de l'œuvre à faire », Bulletin de la Société française de philosophie, 50/1 (1956), p. 4-24 (rééd. SOURIAU, Étienne, Les différents modes d'existence, présenté par I. Stengers et B. Latour, Paris, PUF, 2009, p. 195-217).

24. JAMES, William, Philosophie de l'expérience. Un univers pluraliste, Paris, Les Empêcheurs de penser en rond/Le Seuil, 2007 [1909]. 
25. JAMES, William, The Meaning of Truth, op. cit., p. 226.

26. Titre d'un morceau de Sonny Rollins, sans cesse repris pour parler de l'impro jazz.

27. Voir HENNION, Antoine et MONNIN, Alexandre, «Sous la dictée de l'ange. Enquêter sous le signe d'Étienne Souriau ", Étienne Souriau. Une ontologie de l'instauration, COURTOIS-L'HEUREUX, Fleur et WIAME, Aline (eds), Paris, Vrin, 2015, p. 131-156. Cette relecture d'un auteur difficile doit beaucoup à Alexandre Monnin, le jeune philosophe qui m'avait proposé l'entretien repris dans HENNION, "D'une sociologie de la médiation à une pragmatique des attachements ", art. cit. Outre les chapitres de l'ouvrage précédent, plusieurs textes pénétrants ont récemment été consacrés à Souriau : STENGERS, Isabelle et LATOUR, Bruno, «Le sphinx de l'œuvre », préface à leur réédition des Différents modes d'existence, SOURIAU, Étienne, op. cit., p. 1-75; LAPOUJADE, David, «Étienne Souriau. Une philosophie des existences moindres », DEBAISE, Didier (éd.), Philosophie des possessions, Dijon, Les Presses du Réel, 2011, p. 167-196.

28. Outre Eco (L'œuvre ouverte et Lector in fabula), cité plus haut, voir par exemple ISER, Wolfgang, L'Acte de lecture, Bruxelles, Mardaga, 1985 et JAUSS, Hans R., Pour une esthétique de la réception, Paris, Gallimard, 1978.

29. BOURRIAUD, Nicolas, Esthétique relationnelle, Dijon, Les Presses du réel, 1998 ; MICHAUD, Yves, L'Art à l'état gazeux, Paris, Stock, 2004 ; CHATEAU, Dominique, L'Art comptant pour un, Dijon, Les Presses du réel, 2009 ; FOURMENTRAUX, Jean-Pierre (éd.), L'Ère post-média, Paris, Hermann, 2012. Sur le sujet, voir aussi HENNION, Antoine, « Jouer, interpréter, écouter. Pratiquer la musique, ou la faire agir?», FOURMENTRAUX, Jean-Pierre (éd.), L’Ère post-média, Paris, Hermann, 2012, p. 87-102: une revue critique de l'usage du mot performance.

30. « [L]e trajet complet de l'œuvre, depuis sa première apparition jusqu'à son accomplissement (qui est aliénation totale), ne saurait être considéré comme l'exécution ou l'expression, ou la manifestation progressive, d'un projet original: le croire, ce serait méconnaître et abolir l'efficacité des actes de ce progrès dialectique par questions et réponses » (SOURIAU, Étienne, L'Ombre de Dieu, Paris, PUF, 1955, p. 257).

31. En référence au Saint Matthieu du Caravage représenté sous les traits d'un sténographe affairé à noter sous la dictée de l'ange.

32. HENNION, Antoine, "La mémoire et l'instant. Improvisation sur un thème de Denis Laborde ", Tracés 18 (2010), p. 141-152.

33. HENNION, Antoine, " “Aussi vite que possible..." La virtuosité, une vérité de la performance musicale ? ", Ateliers d'anthropologie du LESC 35, 2011, http://ateliers.revues.org/8764

34. « Le pouvoir et l'acte de se créer soi-même existe, même chez ces êtres qui [...] semblent créés par autrui ; par exemple les œuvres d'art » (SOURIAU, L'Ombre de Dieu, op. cit., p. 279-80). 35. Ibid, p. 215-216.

\section{RÉSUMÉS}

Avec le recul, il me semble que j'ai moins fait de la sociologie de la musique qu'écrit une sociologie depuis la musique. Peut-on, en s'inspirant d'auteurs allant de Michel de Certeau et Louis Marin à William James et Étienne Souriau, reconnaître autrement que sur le mode de la distanciation obligée ou de l'épanchement personnel les rapports constitutifs qu'entretiennent une écriture sur la musique et une pratique de la musique? Les expériences musicales (la création, le jeu, les plaisirs de l'amateur) sont le lieu même de la musique, elles ne sont pas la 
mise en œuvre ou l'appropriation d'un objet extérieur. Dans ces conditions, si la mise par écrit de ces expériences ne peut rendre compte de leur procès, toujours à refaire, en revanche, comme c'est le cas pour l'histoire et la religion chez Certeau, l'écriture contribue à les faire exister autrement, à les prolonger, à les augmenter, tandis que réciproquement, de telles expériences ont soutenu l'écrit du sociologue de la musique que j'ai aussi été.

Pour montrer cela, sur deux situations, je rapprocherai à titre expérimental une écriture et l'expérience qu'elle veut transcrire : l'apprentissage du chant - en m'appuyant sur mon propre cas - et un entretien avec un improvisateur de jazz. La conception de l'improvisation que nous livre ce jazzman fait étonnamment écho à la définition puissante et originale de l'« œuvre à faire » que propose Souriau, sur laquelle conclut ce texte. De telles mises en rapport montrent la nécessité et la possibilité d'une sociologie de l'art qui, loin du scientisme ambiant, se place à la hauteur des œuvres produites, et surtout de ce à quoi elles appellent, des mondes dont elles disent la possibilité. Cet appel de l'œuvre ne concernerait-il pas la sociologie de l'art?

\section{INDEX}

Mots-clés : Musique, écriture, œuvre, amateurs, sociologie de l'art, pluralisme, Certeau, James, Souriau

\section{AUTEUR}

\section{ANTOINE HENNION}

Antoine Hennion (Centre de sociologie de l'innovation, Mines-ParisTech, PSL/CNRS) est sociologue de la musique et de la culture. Au CSI, il a développé une problématisation de la médiation croisant sociologie culturelle et STS. Il a travaillé sur les amateurs, puis l'aide et le care, formes d'attachement qui partagent l'exigence d'une œuvre à faire. Au sein d'un collectif, il développe une pragmatique des attachements (musique, sport, vin, dépendance, migrations...), et travaille sur les questions de méthode et d'engagement que pose une approche pragmatiste de l'enquête en sciences sociales. Auteur de La Passion musicale (2007 [1993]), La Grandeur de Bach (avec J.-M. Fauquet, 2000), Le Vin et l'environnement (avec G. Teil, 2011), il a récemment publié « La question de la tonalité. Relire Lévi-Strauss avec des lunettes pragmatistes? ", L'Année sociologique (2010), « Enquêter sur nos attachements. Comment hériter de William James ? », SociologieS (2015), « “Enfermer Maman !” Le care comme éthique de situation » (avec P. Vidal-Naquet), Sciences sociales et santé (2015), "From ANT to pragmatism: A Journey with Bruno Latour at the CSI", New Literary History (2016). 